\title{
Temporomandibular Joint Prosthesis - 3D-CT Reconstruction before and after Treatment
}

\author{
E. Feltlová1, T. Dostálova1', M. Kašparová1 J. Daněk', P. Hliňáková', M. Hubáček' , J. Nedoma ${ }^{3}$ \\ 'Department of Paediatric Stomatology, Second Faculty of Medicine of Charles University, Prague, Czech Republic \\ 'Department of Mathematics, University of West Bohemia, Plzen, Czech Republic \\ ${ }^{3}$ Institute of Computer Science, Academy of Sciences of the Czech Republic, Prague, Czech
}

Supervisor: Prof. MUDr. Tatjana Dostálová, DrSc., MBA

\section{Summary}

The recent progress in understanding the biomedical basis for temporomandibular joint. (TMJ) therapy as well as in computer and imaging technologies is beginning to provide novel insights into TMJ reconstruction, rational diagnostic and therapeutic strategies. Several methods are available for imaging the TMJ. These include basic radiography (such as panorexes and corrected tomograms), ultrasonography, magnetic resonance imaging, and spiral or conebeam computed tomography. The latter technologies allow the joint to be visualized as sections in different planes and can also be rendered as $3 D$ volumetric reconstructions to enhance diagnostic capabilities. The aim of the study is demonstrate patient's therapy including 1 year recall.

This contribution deals with 3D analysis of the mathematical and computer modeling of biomechanics of the jaw. Case report monitors step by step treatment plan, preprosthetic surgery, mandibular reconstruction and process of healing. 3DCT was used to monitor the patient with dental cysts.

The mathematical and computer TMJ models analysis using overlapping of 3D CT before and after 1 year therapy shows the stability of TMJ partial prosthesis reconstruction.

Mathematical modeling and mathematical simulation of the reconstruction of patient jaw and/or patient TMJ may predict the joint loading of the reconstructed condyle. Our study demonstrated practical use of 3D images during TMJ therapy. Although many of the disorders involving the temporomandibular joint and associated structures can be diagnosed clinically on the basis of the history and physical findings, there are others that require the use of various imaging techniques to make an accurate diagnosis or to determine the extent of involvement. Imaging the hard and soft tissues of the temporomandibular joint can be used not only for treatment planning but also long-term treatment evaluation.

Keywords: dentistry, maxillofacial surgery, partial TMJ prosthesis, threedimensional computed tomography, magnetic resonance imaging, temporomandibular joint

\section{Introduction}

The temporomandibular joint (TMJ) is a bilateral composed joint connecting mandible to the temporal bone. Both joints function bilaterally and any change of one side influences the contra lateral joint. The TMJ has two articulating bone components - mandibular condyle and glenoid fossa of the temporal bone. The condyle has an ellipsoid shape with a mediolateral diameter of $20 \mathrm{~mm}$ and anteroposterior diameter of $10 \mathrm{~mm}$. The long axis of both condyles forms an angle 150-160。. The articular surface is covered with the fibrocartilage, instead of the hyaline cartilage usually present in synovial joints. The fibrocartilage is more resistant to degenerative changes and has better regeneration qualities. Another specialty of TMJ is its disc (discu-sarticularis) of a biconcave shape which fills the space between articular surfaces thus compensating their incongruities. The disc has a unique and complicated composition and it functions as a shock absorber. TMJs function symmetrically and this harmony allows biting, chewing and speaking. Movements are mostly combined together, resulting in the following jaw movements: depression, elevation of mandible (the opening and closing movement), propulsion (the forward movement), and lateropulsion (side movement) [1].
Temporomandibular disorders (TMDs) are a term embracing a number of clinical problems that involve the masticatory musculature, the temporomandibular joint and associated structures, or both. These disorders are accompanied by pain in the masticatory muscles, in the TMJ, and in the associated hard and soft tissues. Other symptoms include limitation or deviation in the mandibular range of motion, TMJ sounds, and/or headaches and facial pain [2]. Symptoms of TMDs occur in approximately 6 to 12 percent of the adult population or approximately ten million individuals in the United States [3]. Many of these patients can be managed with nonsurgical therapies, but some end stage TMJ patients require surgical TMJ repair or reconstruction. The patient can develop end-stage TMJ as a result of trauma, osteoarthritis, reactive arthritis, ankylosis, idiopathic condylar resorption, connective tissue/ autoimmune diseases (e.g. rheumatoid arthritis, psoriatic arthritis, lupus, scleroderma, Sjogren's syndrome, ankylosing spondylitis) or other TMJ pathologies [4]. The joint damage may be followed by insertion of alloplastic implants. Joint replacement is a surgical procedure in which the severely damaged part of the TMJ is removed and replaced by a prosthetic device. If either a condyle or a fossa component of the TMJ is replaced, the surgery is called the partial joint replacement. In total joint replacement, condyle and fossa are both replaced [5]. The recent progress in understanding the biomedical basis for the TMJ therapy as well as in computer and imaging technologies is beginning to provide novel insights into TMJ reconstruction, rational diagnostic and therapeutic strategies. Several methods are available for imaging the TMJ. 
These include basic radiography (such as panorexes and corrected tomograms), ultrasonography, magnetic resonance imaging, and spiral or conebeam computed tomography. The latter technologies allow the joint to be visualized as sections in different planes and can also be rendered as 3D volumetric reconstructions to enhance diagnostic capabilities [6]. The aim of study is demonstrate patient's therapy including 1 year recall.

\section{Case report}

75-year-old patient, suffering from hypercholesterolemia, hypertension and allergy to analgesic Dinyl, was sent to our department from the surgical department of Ceske Budejovice hospital with suspicion of an extensive cyst of the mandible on the right side. From 2002 to 2009 she underwent 3 re-treatments at a private clinic because of an intraoral fistula in the same localization. Among the most important symptoms, which positively responded to antibiotic therapy, were difficult mouth opening, swelling and contracture. Repeated surgeries led to hypoesthesia of the right half of the lower lip. Unsuccessful treatment conduced to CT scan which revealed a large destructive cyst encroaching on the right mandibular ramus from the condyle to the angle of the mandible. During the first ambulatory check up in our clinic visible face asymmetry in the subzygomatic area on the right was clearly visible, the head of the right TM joint was not palpable, mouth opening was not limited, and the oral cavity was without pathological findings. The upper dental arch was toothless and in the lower dental arch teeth 33, 43, 44 were present. Dental rehabilitation in this phase of treatment was done by removable prosthetic appliances. Hypoesthesia of mandibular nerve on the right remained. Due to the extensive destruction of the mandibular ramus and its significant weakness in craniocaudal as well as mediolateral direction, which meant significant risk of pathological fractures, subtotal replacement of right TMJ was proposed (Fig. 1, 2,3).

On June 22, 2009 under general anesthesia extirpation of the cyst of mandible on the right, partial resection of

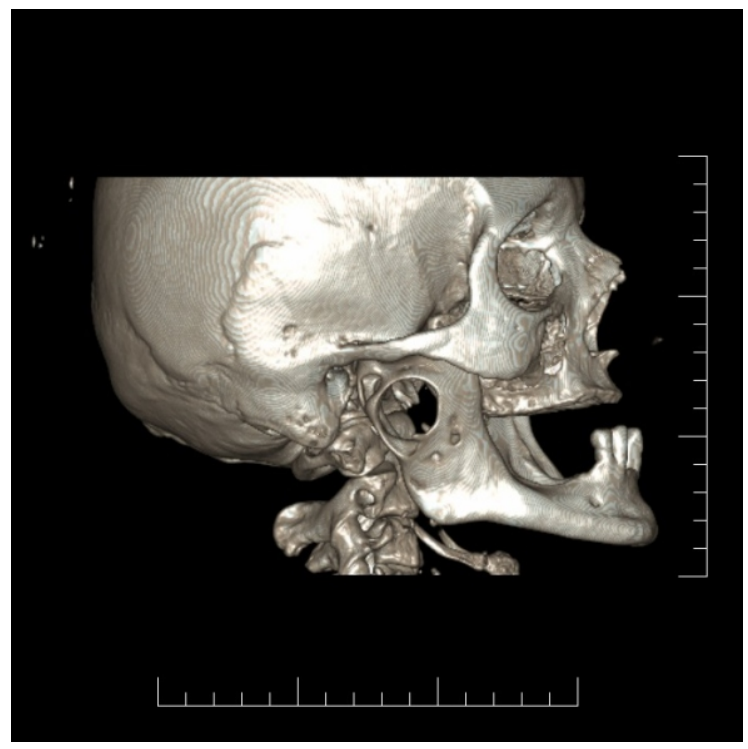

Fig. 1. 3D-CT analysis with destructive cyst in the right ramus mandibulae.

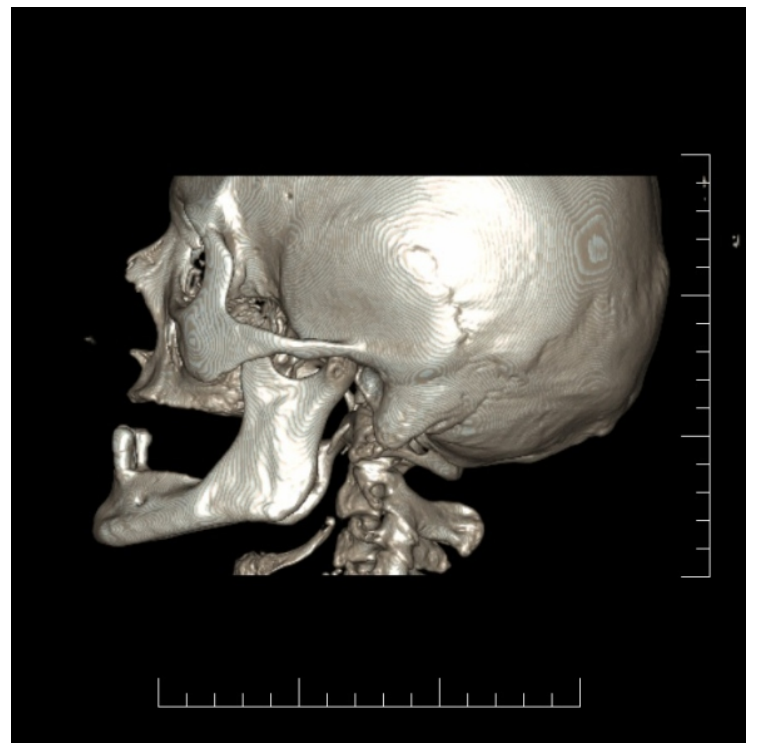

Fig. 2. 3D-CT analysis left healthy TMJ and ramus mandibulae.

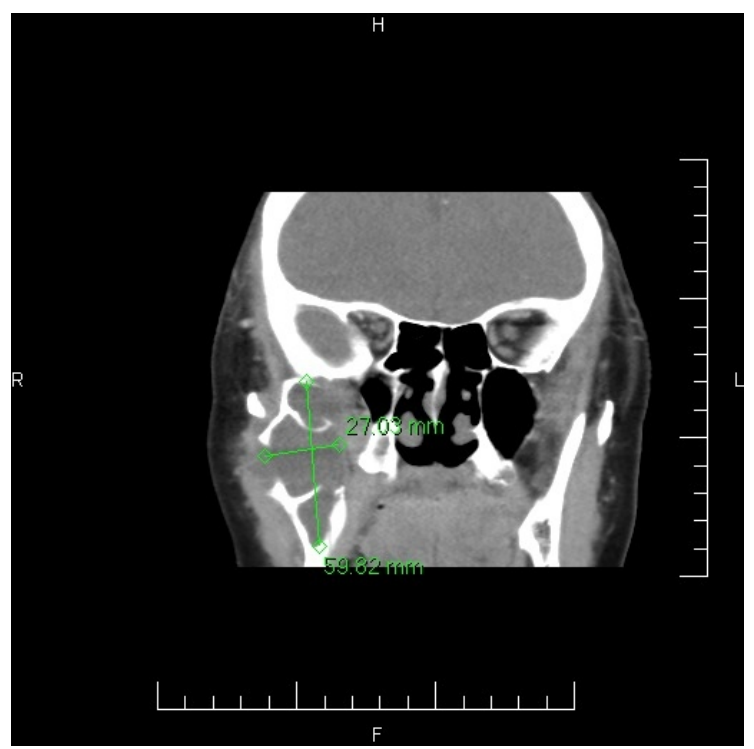

Fig. 3. 3D-CT measurement of cast before therapy. 
the body of mandible and exarticulation of the right TMJ followed by reconstruction of the right TMJ with subtotal replacement (UNI-LOCK) was made. The result of histological examination of biological material taken during the surgery confirmed the diagnosis of a radicular cyst. The postoperative period was covered by antibiotics for a total of 10 days. On the fourth day after the surgery the patient begins to open her mouth, and rehabilitation using wooden spatulas helped her to improve mouth opening. One week after the surgery she was discharged (Fig. 4). After discharge she was followed up in 3-week intervals. The patient was in a good shape, with no swelling, the wounds had healed, her mouth opening gradually improved from $15 \mathrm{~mm}$ to $30 \mathrm{~mm}$, the lower jaw did not turn aside when opening the mouth, palpation of the right TMJ was painless, however mild parenthesis of the mandible nerve persisted. On February 23, 2010 controlled CT scans were made. Results of the last CT scans were encouraging.

Thanks to the 3D-CT visualization of the case, the Surgery was reduced to a minimum, and after 1 year the patient reported minimal discomfort (Fig. 5, 6).

\section{Methods}

The patient data was processed (filtering, cropping, merging, iso-surface extraction, registration, etc.) and visualized using the proprietary software CTVolRegApp, that was developed at the University of West Bohemia under the Multimod Application Framework (MAF) [8], which is a visualisation system based mainly on VTK [7] but also on other specialised libraries. 3D-CT scanner will be used to monitor our patient with dental anomalies. 3D-CT scanners are based on volumetric tomography, using a 2D extended digital array providing an area detector. This is combined with a 3D X-ray beam. The conebeam technique involves a single $360^{\circ}$ scan in which the $x$-ray source and a reciprocating area detector synchronously move around the patient's head, which is stabilized with a head holder. At certain degree intervals, single projection images, known as "basis" images, are acquired.

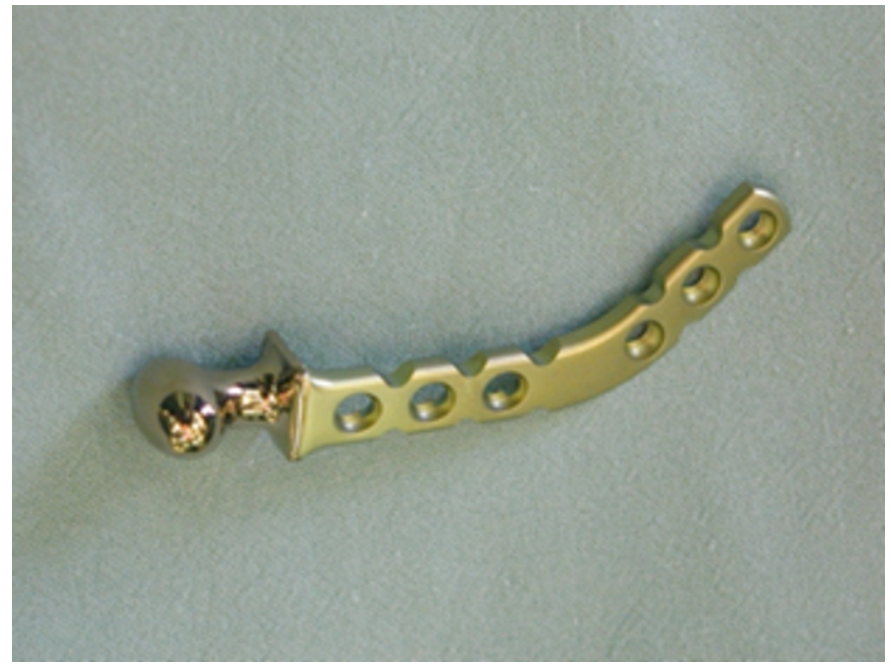

Fig. 4. Partial TMJ prosthesis.

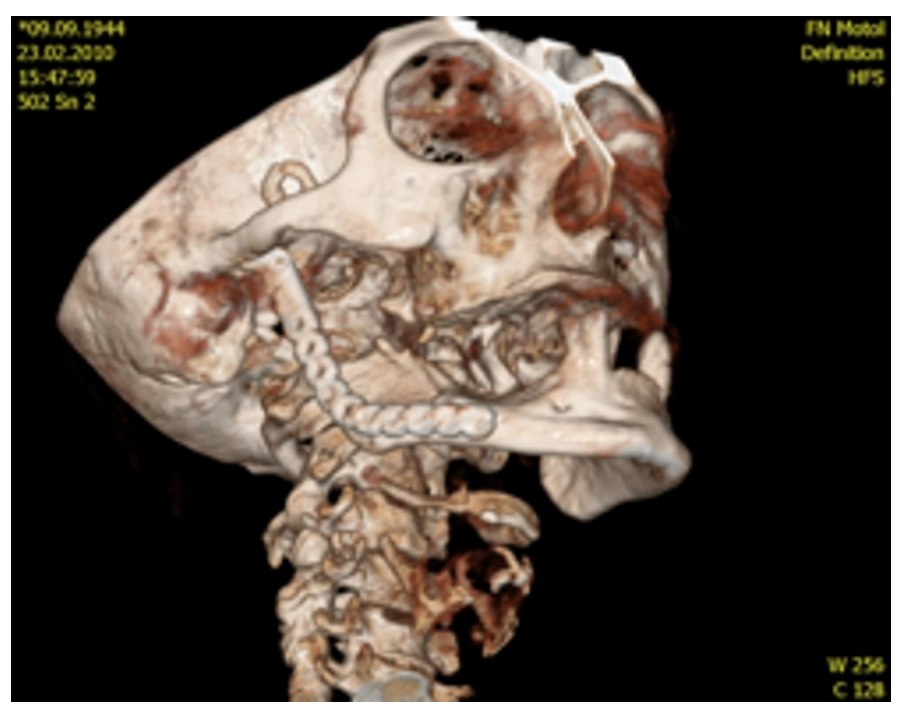

Fig. 5. 3D-CT partial TMJ prosthesis 1 year post insertion.

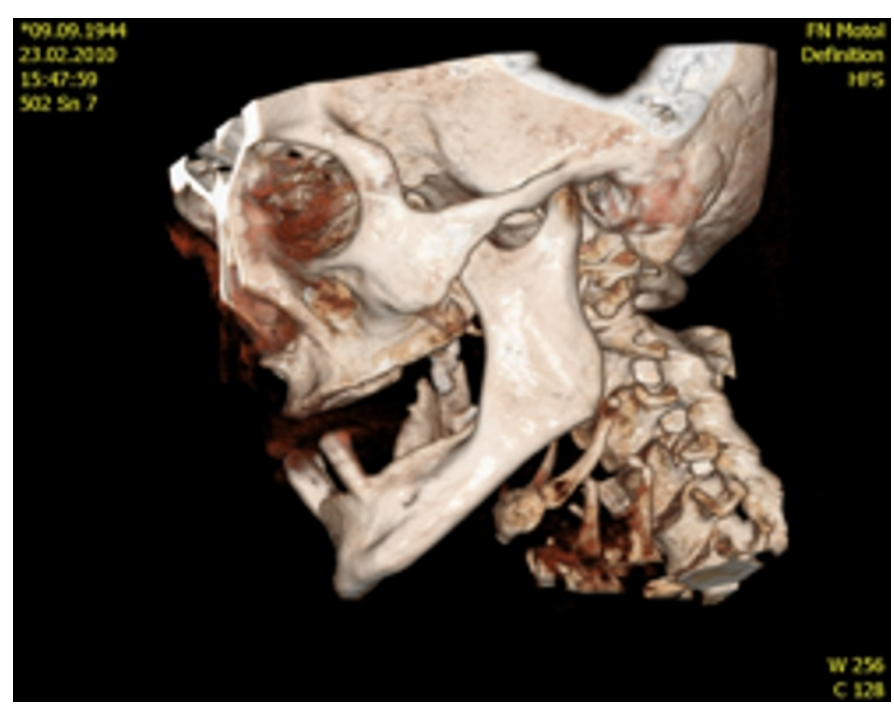

Fig. 6. 3D-CT left healthy TMJ and ramus mandibulae 1 year post insertion. 
These are similar to lateral cephalometric radiographic images, each slightly offset from one another. This series of basis projection images is referred to as the projection data. Software programs incorporating sophisticated algorithms including back-filtered projection are applied to these image data to generate a $3 \mathrm{D}$ volumetric data set, which can be used to provide primary reconstruction images in 3 orthogonal planes (axial, santal and coronal). Although the 3D-CT principle has been in use for almost 2 decades, however, it is only recently, with the development of inexpensive x-ray tubes, high-quality detector systems and powerful personal computers, that affordable systems become commercially available [9].

\section{Results}

Data was organized regarding the presence or absence of bone changes in each view by each observer to allow precise comparison (Fig. 7-10). Fig. 7 shows the damaged right side of the joint, where the large radicular cyst was present. In the area of mandibular ramus and temporomandibular joint destruction of the bone is obvious and was replaced by subtotal prosthesis. Fig. 8 shows the healthy part of mandible. Fig. 9 shows the model of subtotal prosthesis and its fixation in the healthy bone tissue. The prothesis is of the same anatomical shape as the joint. Two medical data sets of TMJ, before and after the treatment, were available for data processing. Each was obtained from various 3D-CT scanners and with a different quality. The position of the head during both CT scans was also different. For comparison of before and after the treatment we imported data sets into software programmers and generated the surface meshes for both models. Consequently the geometric transformations, i. e. translations and rotations, were used to find the best overlap of both geometries. The semitransparent colour was used to depot the original stage before the treatment for better imagination. Comparison of the jaw before and after surgery shows us changes in position in jaws in cranio-facial area.

\section{Discussion}

Mathematical and computer modeling of biomechanics of the jaw and the

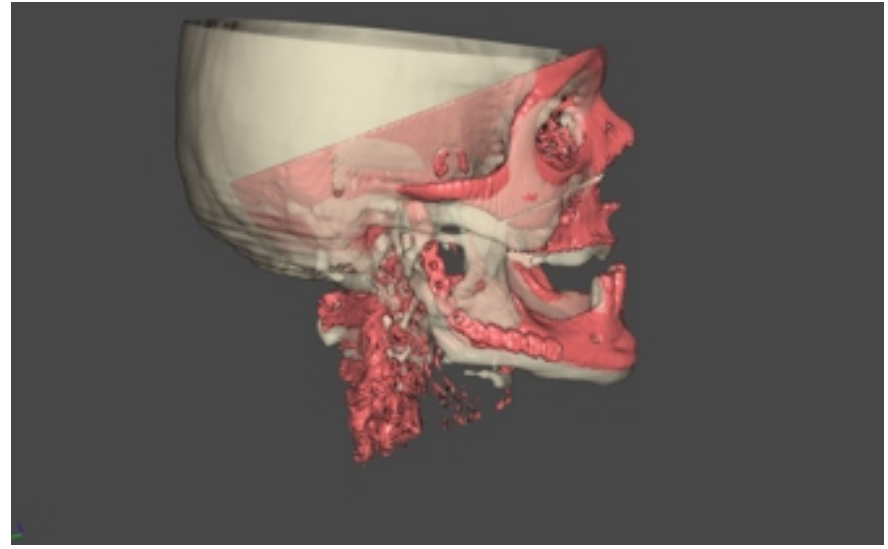

Fig. 7. 3D-CT comparison of the jaw before and after the surgery - right side.

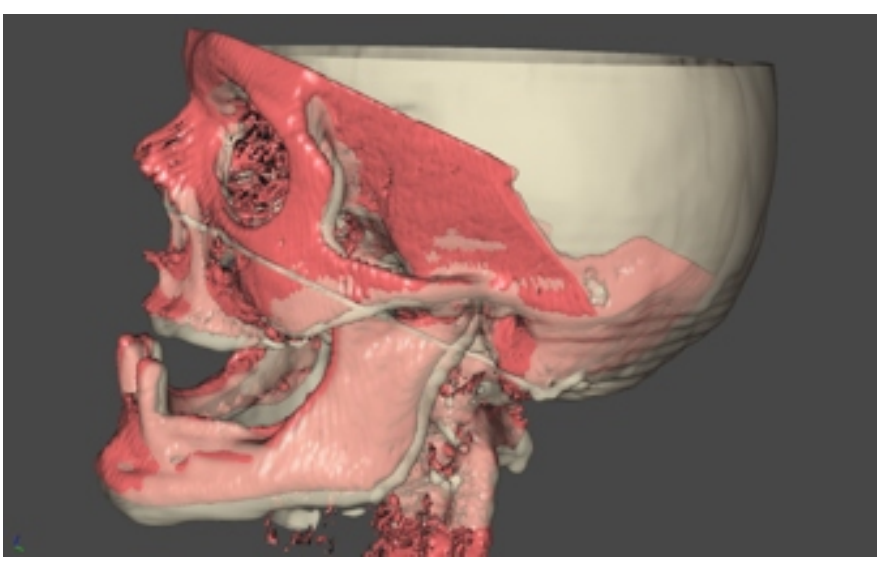

Fig. 8. 3D-CT comparison of the jaw before and after surgery - left side.

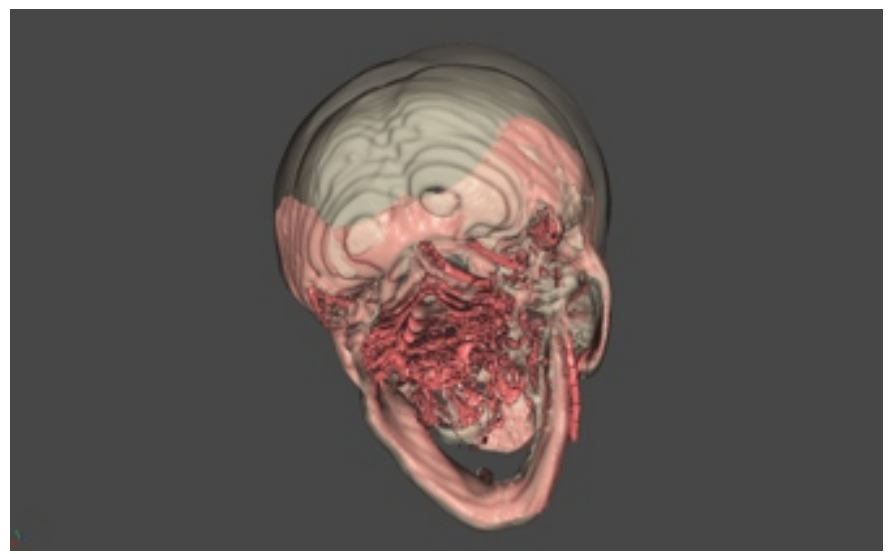

Fig. 9. 3D-CT comparison of the jaw before and after surgery - cranial sight.

temporomandibular joints have the potential for application in many areas of clinical practice, preprosthetic surgery and mandibular reconstruction. Clinical experiences have shown that patients who have had unilateral resection and subsequent reconstruction of the mandible have no pain associated with their reconstructed condyle. Mathematical modeling and mathematical simulation of the reconstruction of patient jaw and/or patient TMJ may predict the joint loading of the reconstructed condyle. The other applications of mathematical modeling of jaw biomechanics can be useful with force changes and changes of their directions 
after orthognathic surgery, and moreover, in evaluation of the influence of occlusal splints on TMJ loading as well as in prediction concerning with the tumor cancers or cysts. The main idea of our previous study continues in this project [10]. The development of CT techniques to acquire, process and manipulate images has improved the knowledge of structures that are otherwise difficult to visualize, such as the TMJ. Standardization of image formats using special protocols, and the use of free software follow a global trend. The manipulation of medical images should be mastered by health professionals who work with diagnosing, planning and treatment of patients, because this technology produces additional images to the ones initially reformatted by radiology technicians [11]. Our study demonstrated practical use of 3D images during the TMJ therapy. Although many of the disorders involving the temporomandibular joint and associated structures can be diagnosed clinically on the basis of the history and physical findings, there are others that require the use of various imaging techniques to make an accurate diagnosis or to determine the extent of involvement. Imaging the hard and soft tissues of the temporomandibular joint can be used not only for treatment planning but also longterm treatment evaluation.

\section{Acknowledgments}

This work was supported by the Grant 9991-3 IGA MZČR, FI-IM5/142 and SVV2010-265513 projects.

\section{References}

[1] Hlinakova P., Dostálová T., Danek J., Nedoma J.: „Temporomandibular joint and its two-dimensional and threedimensional modelling". Mathematics and computers in simulation Vol.80, pp. 1256- 2010, 2010.

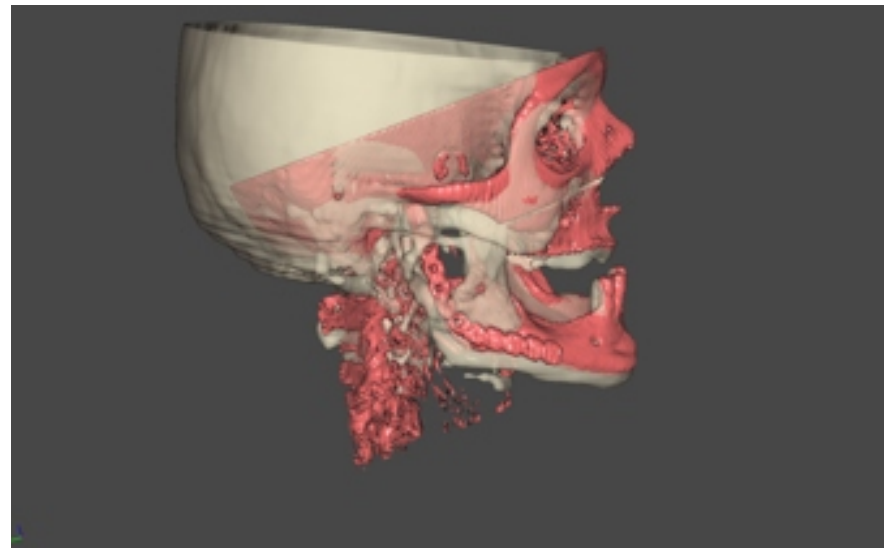

Fig. 10. 3D-CT cross-section after therapy.

[2] Fikáčkova H., Dostálová T., Navrátil L., Klaschka J.: Effectiveness of low-level laser therapy in temporomandibular joint disorders: A placebo-controlled study.

Photomedicine and Laser Surgery.,vol. 25, pp. 297-303, 2007.

[3] Lipton J.A., Ship J.A., Larach-Robinson D. Estimated prevalence and distribution of reported orofacial pain in the United States. J Am Dent Assoc vol. 124, pp. 11521, 1993.

[4] Plesh O., Sinisi S.E., Crawford P. B., Gansky S.A.: Diagnoses based on the research diagnostic criteria for temporomandibular disorders in a biracial population of young women. J Orofac Pain. vol. 19, pp. 6575, 2005.

[5] Wolford L.M., Karras S.C.: Autologous fat transplantation around temporomandibular joint total joint prostheses: preliminary treatment outcomes. J. Oral Maxillofac Surg. vol. 55, pp. 45251, 1997.

[6] Wadhwa S., Kapila S.: TMJ disorders: future innovations in diagnostics and therapeutics. J Dent Educ. vol. 72, pp. 930947, 2008.

[7] Viceconti M., Zannoni C., Testi D., Petrone M., Perticoni S., Quadrani P., Taddei F., Imboden S., Clapworthy G.J. :The Multimod Application Framework: a rapid application development tool for computer aided medicine. Comput Methods Programs Biomed, 85: 138-151, 2007
[8] Schroeder W., Martin K., Lorensen B.: The Visualization Toolkit: an object-oriented approach to 3D graphics. Prentice Hall (1198)

[9] Scarfe W. C.: Clinical applications of conebeam computed tomography in dental practice. J Can Dent Assoc, vol. 72(1), pp. 7580, 2006.

[10] Danek J., Hlinakova P., Preckova P., Dostalova T. , Nedoma J.: Threedimensional modelling of the temporomandibular joints and of their artificial replacements. Lecture Notes in Computer Science (including subseries Lecture Notes in Artificial Intelligence and Lecture Notes in Bioinformatics) 6019 LNCS (PART 4), pp. 62-71, 2010

[11] Farman A.G.: Raising standards: digital interoperability and DICOM. Oral Surg Oral Med Oral Pathol Oral Radiol Endod. vol. 99, pp. 525-6, 2005.

\section{Contact}

MUDr. Eliška Feltlová

Department of Paediatric Stomatology

Second Faculty of Medicine of Charles

University

$\checkmark$ úvalu 84

15006 Prague 5

Czech Republic

e-mail: nelis.elis@seznam.cz 